\title{
Absolute Quantification of Bone Scintigraphy for the Longitudinal Monitoring of Vertebral Fractures With a High-speed Whole-body CZT-SPECT/CT System
}

\section{Achraf BAHLOUL}

CHRU de Nancy: Centre hospitalier regional universitaire de Nancy

\section{Antoine VERGER}

CHRU de Nancy: Centre hospitalier regional universitaire de Nancy

\section{Alain BLUM}

CHRU de Nancy: Centre hospitalier regional universitaire de Nancy

\section{Mohammad Bilal CHAWKI}

CHRU de Nancy: Centre hospitalier regional universitaire de Nancy

\section{Mathieu PERRIN}

CHRU de Nancy: Centre hospitalier regional universitaire de Nancy

\section{Saifeddine MELKHI}

CHRU de Nancy: Centre hospitalier regional universitaire de Nancy

\section{Gilles KARCHER}

CHRU de Nancy: Centre hospitalier regional universitaire de Nancy

\section{Pierre-Yves MARIE}

CHRU de Nancy: Centre hospitalier regional universitaire de Nancy

Laetitia IMBERT ( $\sim$ l.imbert@chu-nancy.fr)

CHRU Nancy

\section{Original research}

Keywords: CZT, SPECT, bone scintigraphy, vertebral fracture, standardized uptake value

Posted Date: May 4th, 2021

DOl: https://doi.org/10.21203/rs.3.rs-442900/v1

License: (1) (i) This work is licensed under a Creative Commons Attribution 4.0 International License.

Read Full License 


\section{Abstract}

This study aimed to determine whether absolute quantification obtained with a high-speed CZT SPECT/CT system provides consistent results on phantom and in bone scintigraphy-based longitudinal monitoring of patients with vertebral fractures and/or fracture cascades.

Materials and Methods. SPECT images were reconstructed as recommended for clinical routine. Both image quality and accuracy of measured activity were evaluated using an IEC body phantom. The optimized reconstruction process was applied to routine $\leq 15 \mathrm{~min}{ }^{99} \mathrm{~m}$ Tc-HDP SPECT spine recordings, which had been previously acquired from 25 patients ( $74 \pm 12$ years old) at both early ( $1.3 \pm 1.1$ months) and late (5.2 \pm 2.3 months) stage assessments of an acute vertebral fracture of a traumatic and/or osteoporotic origin.

Results. A SPECT reconstruction with 32 equivalent iterations was used based on high levels of foci detectability in spheres as small as $0.6 \mathrm{~mL}$ in volume, and accuracy of measured activity, although the latter was affected by partial volume effect for spheres $\leq 5.8 \mathrm{~mL}$.

SUVmax from patients' intact T1 vertebrae, used as a reference, remained stable between $1^{\text {st }}$ and $2^{\text {nd }}$ SPECT recordings $(5.7 \pm 1.1$ vs. $5.8 \pm 1.1, p=0.76)$. SUVmax from the initially fractured vertebrae were 3 -fold higher on $1^{\text {st }}$ SPECT $(21.0 \pm 8.5, p<0.001$ vs. T1 SUVmax $)$ and markedly decreased on $2^{\text {nd }}$ SPECT $\left(11.2 \pm 4.2, p<0.001\right.$ vs. $1^{\text {st }}$ SPECT). Inverse changes in SUVmax were documented for newly compacted fractures apparent on the $2^{\text {nd }}$ SPECT $\left(1^{\text {st }}\right.$ SPECT: $7.4 \pm 2.0$ vs. $2^{\text {nd }}$ SPECT: $\left.21.8 \pm 10.3, p=0.002\right)$ together with an increased bone density on CT (in mean Hounsfield Units: $121 \pm 28$ vs. $190 \pm 39, p=0.003$ ). Rate of SUVmax > 7.5 was $98 \%(56 / 57)$ for measurements obtained in vertebrae fractured in the preceding 7months, whereas this rate was only $4 \%$ for the reference intact vertebrae $(2 / 50)$.

Conclusion. High-speed recordings with this CZT-SPECT/CT system provide reliable SUV measurements that may attest to longitudinal changes in vertebral bone metabolism and especially changes related to fracture healing or recurrence.

\section{Introduction}

Several recently published studies have given evidence of a significant contribution of absolute quantification in the interpretation of bone SPECT images, especially for the monitoring of longitudinal changes after treatment [1-3], and also for the characterization of metastasis [4-6], osteomyelitis, osteonecrosis [7], and certain degenerative bone lesions [8, 9]. To the best of our knowledge, however, none of these previous quantitative bone studies were planned with new high-speed CZT cameras, nor conducted in patients with bone fractures.

Bone scintigraphy is increasingly used in the pre-therapeutic assessment and monitoring of patients with compression and/or traumatism of vertebrae at a possible multi-site level [10-13], with the main objective being to select the painful lesions for which a vertebral augmentation procedure, i.e., 
vertebroplasty or kyphoplasty, would be beneficial. Such fractures may occur in complex settings, within ankylosed thoracolumbar spinal segments [14] and especially, during a downward spiral of osteoporosisrelated recurrent fractures (known as the vertebral fracture cascade) that may be favored by a vertebral augmentation [15]. However, none of the previously documented bone scintigraphy studies of patients with compression and/or fracture was analyzed using an objective absolute method for quantification, nor conducted with a new high-speed CZT camera. Such cameras have the advantages of not only enhancing image quality, but also of reducing the recording time $[16,17]$, a property that is particularly advantageous in patients with painful fractures.

The whole-body Veriton ${ }^{\circledR}$ camera (Spectrum Dynamics Medical, Caesarea, Israel) combines CZT detectors with an original $360^{\circ}$ ring-configuration geometry that allows the detectors to be positioned very close to the patient, a configuration that is likely to maximize both count sensitivity and image quality [17-19]. With this camera, it has already been demonstrated that a whole-body 3-dimensional SPECT recording may be obtained in no more than 20 minutes for bone ${ }^{99 \mathrm{~m}} \mathrm{Tc}$-scintigraphy [17], as well as for lutetium-177 therapeutic imaging [18]. Absolute quantification has also been developed with this camera and whilst the quantification of lutetium-177 has been investigated for dosimetry [18], as yet there has not been any assessment for routine bone scintigraphy.

This study aimed to determine whether absolute quantification obtained with this high-speed CZT SPECT/CT system provides consistent results on phantom and in bone scintigraphy-based longitudinal monitoring of patients with vertebral fractures and/or fracture cascades.

\section{Materials And Methods}

All images were acquired on the Veriton ${ }^{\circledR}$ hybrid system comprising the high-sensitivity $360^{\circ} \mathrm{CZT}$-camera and 64-row detector CT. The present study involves the analysis of a routine protocol for bone SPECT/CT recording applied: (i) on a phantom for optimizing the image reconstruction process, with respect to image quality and absolute quantification measurement, and (ii) in patients with acutely fractured vertebrae to check for the consistency of SUV measurements, and especially for changes over time in fractured vs. non-fractured vertebrae.

The study was approved on January 22nd, 2021 by the Ethics Committee of the CHRU of Nancy (reference number 297). This research complied with the principles of the Declaration of Helsinki. Informed consent was obtained from all individuals included in the study.

\section{Patients' bone SPECT/CT recordings}

Twenty-five consecutive patients were retrospectively selected on the basis of prior imaging on the Veriton ${ }^{\circledR}$ hybrid system, comprising: a 1st SPECT/CT for pre-therapeutic assessment prior to a possible vertebral augmentation procedure after an acute vertebral fracture of traumatic and/or osteoporotic 
origin, and a 2nd SPECT/CT at a later stage, mainly due to suspicion of new vertebral fractures (vertebral fracture cascade). The fracture date was considered to be that of the acute onset or worsening of pain.

SPECT/CT recordings commenced 3 to 4 hours after the injection of approximately $550 \mathrm{MBq}$ of ${ }^{99 \mathrm{~m}} \mathrm{TC}-$ hydroxydiphosphonate (HDP). CT was recorded first, with the following parameters: $120 \mathrm{kV}, 150 \mathrm{mAs}$ modulation, pitch of 0.8 , slice thickness of $1.25 \mathrm{~mm}$, increment $1.25 \mathrm{~mm}$, iterative reconstruction, bone filter. SPECT was recorded thereafter with an energy window of $140 \mathrm{keV} \pm 7.5 \%$ during a total recording time of 10 to 15 mins ( 2 to 3 bed positions of 5 mins each).

\section{Phantom experiments}

The CZT-camera was previously calibrated with the clinical routine reconstruction parameters and by using a cylinder filled-in with a homogeneous solution of ${ }^{99 \mathrm{~m}} \mathrm{Tc}$, as previously described [20].

A NEMA IEC body phantom (Data Spectrum Corporation, Durham, NC, USA) was filled-in within the background compartment using a ${ }^{99 \mathrm{~m}} \mathrm{Tc}$ solution with activity concentration of $16,50 \mathrm{kBq} \cdot \mathrm{mL}^{-1}$ at scan time; and with an 8.3-fold higher activity concentration $\left(137,05 \mathrm{kBq} \cdot \mathrm{mL}^{-1}\right)$ within six spherical inserts having respective volumes of $0.6,1.1,2.6,5.6,11.5$ and $26.5 \mathrm{~mL}$. This phantom was recorded at the center of the camera field of view using the routine protocol recommended for bone scintigraphy, although with recording time reduced to 2 minutes in a single bed position. Accordingly, the total number of recorded counts was in the range observed for the thoraco-abdominal bed positions of our routine bone scintigraphy recordings (1.7 Mcounts).

\section{Image reconstructions and analysis}

SPECT images of the IEC phantom were reconstructed using two OSEM iterative methods both being recommended by the manufacturer for optimizing the image quality of bone SPECT. The first one, which provides the best spatial resolution but requires high level of count statistics, involves 8 subsets, 8 iterations and a kernel inter-iteration filter with a factor 0.125 . The second one, which favors contrast-tonoise ratio and is more adapted to lower count statistics, involves 8 subsets, 4 iterations and a factor 0.2 for a kernel inter-iteration filter. The remaining parameters were fixed as follows: post-reconstruction median filter ( $3 \times 3 \times 3$ voxels); and corrections for scatter, attenuation with the CT attenuation map, resolution recovery with a point-spread function, and partial volume with a dedicated vendor-proprietary algorithm (PVC) that uses the anatomical information provided by the CT scan. For the phantom images, this PVC algorithm was applied on a CT scan for which the spheres' density was fixed at 700 Hounsfield Units (HU), i.e., an intermediate level between the HU from spongious and cortical bone. All SPECT images were displayed through cubic voxels of $2.46 \times 2.46 \times 2.46 \mathrm{~mm}^{3}$.

MIM software (MIM Software Inc, Cleveland, OH, USA) was used to provide paired display of SPECT and CT images. Volumes-of-interest (VOIs) were drawn manually, encompassing: 1) each of the 6 spheres of the IEC phantom, and 2) the bodies of targeted fractured vertebrae and the reference T1 vertebra on each patient's SPECT/CT exam. The targeted fractured vertebrae were defined as those showing the highest SUV values on SPECT recordings, together with typical CT signs of bone compression and/or fracture. In 
addition, the T1 vertebra was used as a control reference since this vertebra is at very low risk of osteoporotic fractures (21), and no fracture or severe arthrosis lesion was detected on T1 vertebra on any of our SPECT/CT recordings. Additionally, cement volume was excluded from the VOls of all vertebrae treated by cementoplasty.

Mean HU were obtained from the CT vertebral VOIs; maximal SUV (SUVmax) from the SPECT vertebral VOIs, and a maximal activity concentration expressed in \% of the actual concentrations was computed with the SPECT phantom VOIs. SUV were determined with the following formula: $A_{V O I} \times B W / A_{I N J}$, where $A_{V O I}$ is the activity concentration within a $\mathrm{VOI}$ in $\mathrm{kBq} / \mathrm{ml}, \mathrm{A}_{\mathrm{INJ}}$ is the decay-corrected injected activity in $\mathrm{kBq}$, and $\mathrm{BW}$ is the patient body weight in $\mathrm{g}$.

A contrast-to-noise ratio $(\mathrm{C} / \mathrm{N})$ was computed on each SPECT-image reconstruction of the IEC phantom, with a spherical VOI of $37 \mathrm{~mm}$ diameter corresponding to the diameter of the largest sphere. This VOI was placed at the center of the largest sphere to measure mean sphere activity ("Mean sphere" in counts per second) and corresponding standard deviation ("SD sphere"). A $5 \mathrm{~cm}$ translation of the VOI was subsequently applied to measure mean background activity ("Mean BKG" in counts per second) and corresponding standard deviation ("SD BKG"). Contrast-to-noise ratio was finally computed with the

following equation [19]: $\frac{\text { Mean sphere-Mean } B K G}{\sqrt{S D \text { sphere }{ }^{2}+S D B K G^{2}}}$.

\section{Statistical analysis}

Qualitative variables were expressed with frequencies and quantitative values, with means \pm standard deviations. Mann-Whitney tests and Wilcoxon signed-rank tests were used respectively for the unpaired and paired comparisons of quantitative variables. For all tests, a p value $<0.05$ was considered as indicative of a significant difference. This statistical analysis was performed with a SPSS 25.0 software (IBM Corp. ${ }^{\circledR}$, Armonk, NY, USA).

\section{Results}

\section{Phantom experiments}

Mean background activity ranged between 98 and $99 \%$ of the actual activity concentration when measured on images provided by each of the two reconstructed methods.

In addition, as detailed in Fig. 1, the maximal activity concentrations measured on phantom spheres exhibited an increase according to the sphere size and was clearly underestimated for the smallest spheres with volume $\leq 5.6 \mathrm{~mL}$. This underestimation was somewhat more marked for the reconstruction favoring signal-to-noise ratio at the expense of spatial resolution, but this latter had the advantage of improving image quality and facilitating image analysis on both phantom and patient bone SPECT images (Fig. 1). Thereby, the reconstruction method favoring contrast-to-noise ratio was considered to 
provide the best compromise and robustness for routine image interpretation and it was selected for the additional analyses (Fig. 1).

\section{Characteristics of the study population}

At the time of the 1 st SPECT/CT, mean age of the study patients was $73 \pm 12$ years (51 to 90 years), 11 (44\%) were women, and $15(60 \%)$ had a previous history of vertebral compaction fracture and could thus be considered to be affected by a vertebral fracture cascade. An at least partially osteoporotic mechanism was suspected for all these acute fractures except in 4 patients for whom this mechanism was presumed purely traumatic.

The 1st SPECT/CT was performed at a mean of $1.3 \pm 1.1$ months after the acute fracture date. It was prescribed as part of a pre-therapeutic evaluation based on which 12 patients were referred for cementoplasty and 13 referred for conservative treatment involving a back brace.

The 2nd SPECT/CT was performed at a mean of $5.2 \pm 2.3$ months after the initial acute fracture episode and it was prescribed for the persistence or resurgence of back pain in all patients. A new episode of acute vertebral fracture was finally diagnosed in 12 patients for whom the mean delay-time between symptom resurgence or aggravation and the 2 nd SPECT was $4.9 \pm 4.1$ weeks.

Overall a total of 37 vertebrae were considered as acutely fractured, based on evocative symptoms and analysis of both the 1st and 2nd SPECT/CT. In order of decreasing frequency, these vertebral fractures were located on L2 in 7 cases, L1 and T12 in 5 each, T11 and L4 in 4 each, T7 in 3 cases, T4, T10, and L3 in 2 each, and T5, T6 and L5 in one case each.

\section{Results of SPECT/CT imaging}

As detailed in Table 1, SUVmax measurements from the reference intact T1 vertebrae remained stable between the 1 st and 2 nd SPECT ( $5.7 \pm 1.1$ vs. $5.8 \pm 1.1, p=0.76)$. SUVmax from the initially fractured vertebrae were 3-fold higher on 1st SPECT $(21.0 \pm 8.5, p<0.001$ vs. T1 SUVmax) and markedly decreased on 2 nd SPECT $(11.2 \pm 4.2, p<0.001$ vs. 1 st SPECT $)$. 
Table 1

Paired comparisons between the 1st and 2nd SPECT/CT of imaging parameters recorded on 25 initially fractured vertebrae and the 25 reference non-fractured T1 vertebrae, as well as for 12 vertebrae identified as newly fractured on the 2nd SPECT.

\begin{tabular}{|c|c|c|c|}
\hline & 1st SPECT/CT & 2nd SPECT/CT & $P$ value \\
\hline \multicolumn{4}{|c|}{ Reference T1 vertebrae $(n=25)$} \\
\hline SUVmax & $5.7 \pm 1.1$ & $5.8 \pm 1.1$ & 0.76 \\
\hline Mean HU & $178 \pm 32$ & $173 \pm 31$ & 0.22 \\
\hline \multicolumn{4}{|c|}{ Initially fractured vertebrae $\left(n=25^{\star}\right)$} \\
\hline SUVmax & $21.0 \pm 8.5$ & $11.2 \pm 4.2$ & $<0.001$ \\
\hline Mean HU & $232 \pm 54$ & $232 \pm 59$ & 0.71 \\
\hline \multicolumn{4}{|c|}{ Newly fractured vertebrae $\left(n=12^{\star}\right)$} \\
\hline SUVmax & $7.4 \pm 2.0$ & $21.8 \pm 10.3$ & 0.002 \\
\hline Mean HU & $121 \pm 28$ & $190 \pm 39$ & 0.003 \\
\hline
\end{tabular}

On average in the overall population, the difference in SUVmax from the initially fractured vertebrae between 1 st and 2 nd SPECT was $9.7 \pm 8.7$, and this difference was comparable between the 12 patients for whom this vertebra was treated by cementoplasty $(8.8 \pm 9.9)$ and the 13 other patients who had conservative treatment $(10.6 \pm 7.7, p=0.23)$.

Broad differences in SUVmax were also documented on the 12 vertebrae showing a new acute fracture between the 1st and 2nd SPECT/CT (1st SPECT: $7.4 \pm 2.0$ vs. 2nd SPECT: $21.8 \pm 10.3, p=0.002$ ). These differences were associated with an increase in mean $\mathrm{HU}$ and thus, in bone density, between the 1 st and the 2 nd SPECT/CT (121 $\pm 28 \mathrm{HU}$ vs. $190 \pm 39 \mathrm{HU}, \mathrm{p}=0.003)$. By contrast, mean HU were stable between the 1st and 2nd CT for T1 as well as for the initially fractured vertebrae (Table 1).

All SUVmax measured on T1 and on the acutely fractured vertebrae are displayed according to the delaytime from the fracture date in Fig. 2. This Figure shows a decline over time in the SUVmax from fractured vertebrae, but not for the SUVmax from T1. It may additionally be observed that SUVmax was $>7.5$ in as many as $98 \%$ (56/57) of measurements obtained on vertebrae fractured in the preceding 7 -months, whereas rate of SUVmax $>7.5$ was only $4 \%$ for reference intact vertebrae $(2 / 50)$.

These results are illustrated in Fig. 3 by images from the same patient with color and grey scales similarly adjusted to a maximal SUV of 13 on the 1 st and 2 nd SPECT. This likely leads to facilitate comparison 
between the two SPECTs and to favor the identification of bone structures reaching a $\geq 7.5$ SUV level (i.e., orange for the color scale and dark grey for the gray scale). In particular, the initially fractured vertebra remains easily detectable on the 2 nd SPECT, despite a marked decreased uptake from the 1st SPECT. Opposingly, an increased uptake may be easily detected between the 1 st and 2nd SPECT on several vertebrae showing CT signs of new compressions on the 2nd SPECT/CT, in a typical setting of vertebral cascade fracture.

\section{Discussion}

This study shows that in conditions of image reconstruction targeting a high level of image quality, highspeed recording from this whole-body $360^{\circ} \mathrm{CZT}$-SPECT/CT system provides reliable and potentially helpful SUV measurements in patients with vertebral fractures and/or compaction.

However, the poorer spatial resolution of SPECT compared with PET, and resultant higher partial volume effect, is considered as a disadvantage for SUV measurement. In analysis of the present study, only one SPECT reconstruction series was utilized with parameters favoring contrast-to-noise ratio -i.e., cubic voxels of only $2.46 \mathrm{~mm}$ and partial volume correction - and also, with filtering -i.e., median and kernel filters and only 32 equivalent OSEM iterations. These reconstruction parameters were those considered to provide the best compromise between image quality and the accuracy of activity measurements - i.e. high foci detectability, even for a $0.6 \mathrm{~mL}$ sphere volume, and with significant SUVmax underestimation only for spheres $\leq 5.8 \mathrm{~mL}$ (Fig. 1).

SUVmax was preferred here to other SUV parameters such as SUV mean and SUV peak. SUV mean was difficult to use in the absence of any precise knowledge regarding limits of the diseased bone volumes and when applied to the entire bodies of vertebrae, its ability to separate fractured from intact vertebrae was lower than that of SUVmax (see supplemental Fig. 4). In addition, a $1 \mathrm{~cm}^{3}$ volume within which the SUV peak is commonly determined was too large, exceeding the thickness of several very flattened vertebrae and the volume of the smallest phantom sphere. Finally, the well-known confusing influence of noise level on the determination of SUVmax $[23,24]$ was minimized here, due to our choice of reconstruction parameters favoring the contrast-to-noise ratio of the SPECT images.

Overall, our phantom results agree with the conclusions achieved by previous comparable studies planned on more conventional gamma-cameras, regarding the acceptable accuracy of SPECT SUV measurements [25, 26], especially for volumes $\geq 10 \mathrm{~mL}$ [27] and when associated with CT-based methods enabling reduction in the partial volume effect [28-30]. It is of note however, that underestimation of SUVmax for spheres $<10 \mathrm{~mL}$ was no longer observed when utilizing alternative reconstruction parameters that favored spatial resolution to a greater extent ( $\geq 20$ OSEM equivalent iterations, less image filtering), albeit at the cost of an increase in the noise level (results not shown).

However, the remaining partial volume effects, observed here for volumes smaller than 5 to $10 \mathrm{~mL}$, constitute a limitation, considering that the diseased parts of certain compacted vertebrae may represent 
a smaller than 5 to $10 \mathrm{~mL}$ volume. Despite this limitation, however, consistent results were observed in our longitudinal study of patients with vertebral fractures and/or compactions, thereby reinforcing the potential usefulness of our method of SUV measurement. These results include the stability over time of the SUVmax from intact control reference vertebrae. The T1 vertebrae were chosen for this stability analysis because of their very low risk of osteoporotic fractures [21], and because they were visually intact on the SPECT/CT images of our all patients.

The results also demonstrate a dramatic decrease over time for the SUVmax from fractured vertebrae, as would be expected, although elevated SUVmax levels were still documented during a 7-month period (Fig. 3). Such persistence of elevated SUVmax over the longer-term is not surprising considering that a great proportion of bone scintigraphy remains visually abnormal even at one year after a vertebral compression fracture [31].

SUV were already proven helpful for documenting longitudinal changes with PET imaging and more recently with bone-SPECT [1-3]. However, to the best of our knowledge, the present study is the first to use SUV for analyzing longitudinal changes in the metabolism of bone fractures. The potential usefulness of SUV measurement in this setting is best illustrated in Fig. 3 by the easy identification of any increase in bone metabolism, corresponding to new compaction fractures involved in a vertebral fracture cascade, when serial SPECT images are displayed with the same SUV-based scaling.

Furthermore, this assessment was obtained with recording times of only 12 to 15 minutes for the entire spine. This latter property is likely advantageous in patients for whom a prolonged supine position on the camera bed is difficult to endure due to their painful fractures.

An additional observation was that as many as $98 \%$ of the fractured vertebrae exhibited SUVmax $>7.5$ in the 7-month period following the acute episode, whereas this was highly unusual in the reference intact T1 vertebrae with only $4 \%$ exhibiting SUVmax $>7.5$. Therefore, it may be considered that an image scaling favoring the identification of bone structures reaching a $>7.5 \mathrm{SUV}$ level is required to easily detect most bone fractures, and especially the oldest ones.

Until now, this image scaling has routinely been done in an empirical and subjective visual way, mainly based on the \% of maximal voxel activity, with high reader variability being a difficulty for uniformity of SPECT diagnoses. Standardized SUV-based scaling would likely enhance bone SPECT analysis reproducibility by optimally standardizing readers' diagnostic review. It might also alleviate the difficulties encountered when scaling images where bone lesions are very diffuse (i.e., a super scan) or have very high maximal activity levels. This point is illustrated in a supplemental Fig. 5 by the SPECT images from an actual clinical case where the diagnosis could be corrected with a secondary analysis, thanks to the application of an SUV-based scaling method.

In conclusion, the high-speed whole-body CZT-SPECT/CT system presented here, in association with an adapted SPECT reconstruction for bone scintigraphy images, provides high image-quality together with reliable SUV measurements, at least for structures with $\geq 5$ to $10 \mathrm{~mL}$ volume. These SUV measurements may attest to the longitudinal changes in vertebral bone metabolism -i.e., SUV decrease during fracture 
healing and iSUV increase after a fracture recurrence and within a possible framework of cascade fractures. And further, more generally, these SUV measurements might also help to enhance the reproducibility and robustness of bone scintigraphy analyses.

\section{Declarations}

\section{Funding}

None

\section{Ethics approval and consent to participate}

All procedures performed in studies involving human participants were in accordance with the 1964 Helsinki declaration and its later amendments or comparable ethical standards. The study was approved on January 22 ${ }^{\text {nd }}, 2021$ by the Ethics Committee of the CHRU of Nancy (reference number 297). Informed consent was obtained from all individuals included in the study.

\section{Consent for publication}

Not applicable

\section{Availability of data and material}

The data that support the findings of this study are available on request from the corresponding author (LI).

\section{Conflicts of interest/competing interests}

The authors declare no direct conflict of interest, however note that our center is used by Spectrum Dynamics to conduct site visits for demonstrations of the Veriton camera.

\section{Authors' contributions}

All authors contributed significantly to the analysis and interpretation of the data $(A B, A V, A B, M C, M P, S F$, $G K, P Y, L I)$, to the writing of the manuscript (AB, PY, $L I)$ and to the revision of the manuscript $(A B, A V, A B$, PY, LI).

\section{Acknowledgements}

The authors wish to thank Justine McQuillan for critical review of the manuscript.

\section{References}

1. Kitajima K, Futani H, Tsuchitani T, Takahashi Y, Yamakado K. Quantitative Bone Single Photon Emission Computed Tomography/Computed Tomography for Evaluating Response to 
Bisphosphonate Treatment in Patients with Paget's Disease of Bone. Case Rep Oncol. 2020;13:82934.

2. de Laroche R, Bourhis D, Robin P, et al. Feasibility Study and Preliminary Results of Prognostic Value of Bone SPECT-CT Quantitative Indices for the Response Assessment of Bone Metastatic Prostate Carcinoma to Abiraterone. Front Med (Lausanne). 2020;6:342.

3. Schmidkonz C, Atzinger A, Goetz TI, et al. 99mTc-MIP-1404 SPECT/CT for Patients With Metastatic Prostate Cancer: Interobserver and Intraobserver Variability in Treatment-Related Longitudinal Tracer Uptake Assessments of Prostate-Specific Membrane Antigen-Positive Lesions. Clin Nucl Med. 2020;45:105-12.

4. Motegi K, Matsutomo N, Yamamoto T, Koizumi M. Evaluation of bone metastasis burden as an imaging biomarker by quantitative single-photon emission computed tomography/computed tomography for assessing prostate cancer with bone metastasis: a phantom and clinical study. Radiol Phys Technol. 2020;13:219-29.

5. Tabotta F, Jreige M, Schaefer N, Becce F, Prior JO, Nicod Lalonde M. Quantitative bone SPECT/CT: high specificity for identification of prostate cancer bone metastases. BMC Musculoskelet Disord. 2019;20:619.

6. Kuji I, Yamane T, Seto A, Yasumizu Y, Shirotake S, Oyama M. Skeletal standardized uptake values obtained by quantitative SPECT/CT as an osteoblastic biomarker for the discrimination of active bone metastasis in prostate cancer. Eur J Hybrid Imaging. 2017;1:2.

7. Hata H, Kitao T, Sato J, et al. Quantitative bone single photon emission computed tomography analysis of the effects of duration of bisphosphonate administration on the parietal bone. Sci Rep. 2020;10:17461.

8. Suh MS, Lee WW, Kim Y-K, Yun P-Y, Kim SE. Maximum Standardized Uptake Value of (99m)Tc Hydroxymethylene Diphosphonate SPECT/CT for the Evaluation of Temporomandibular Joint Disorder. Radiology. 2016;280:890-896.

9. Kim J, Lee H-H, Kang Y, et al. Maximum standardised uptake value of quantitative bone SPECT/CT in patients with medial compartment osteoarthritis of the knee. Clin Radiol. 2017;72:580-9.

10. Martin JG, Goldman DT, Dabrowiecki AM, Newsome J, Bercu ZL, Gilliland C. Additional Magnetic Resonance or Nuclear Scintigraphy Imaging Influences Approach to Vertebral Augmentation: A Single Institution Experience. Spine (Phila Pa 1976). 2020;45:E927-32.

11. Okazaki T, Nakagawa H, Yagi K, Hayase H, Nagahiro S, Saito K. Bone scintigraphy for the diagnosis of the responsible level of osteoporotic vertebral compression fractures in percutaneous balloon kyphoplasty. Clin Neurol Neurosurg. 2017;152:23-7.

12. Zhao Q-M, Gu X-F, Liu Z-T, Cheng L. The Value of Radionuclide Bone Imaging in Defining Fresh Fractures Among Osteoporotic Vertebral Compression Fractures. J Craniofac Surg. 2016;27:745-8.

13. Tang Z, Lei Z, Yang H, Chen K. Value of bone scan imaging in determining painful vertebrae of osteoporotic vertebral compression fractures patients with contraindications to MRI. Orthop Surg. 2012;4:172-6. 
14. Prestat AJ, Gondim Teixeira PA, Rauch A, et al. First intention vertebroplasty in fractures within an ankylosed thoracolumbar spinal segment. Diagn Interv Imaging. 2021 Feb 3:S2211-5684(21)000292.

15. Jacobson RE, Palea O, Granville M. Progression of Vertebral Compression Fractures After Previous Vertebral Augmentation: Technical Reasons for Recurrent Fractures in a Previously Treated Vertebra. Cureus. 2017 Oct 16;9(10):e1776.

16. Yamane T, Kondo A, Takahashi M, et al. Ultrafast bone scintigraphy scan for detecting bone metastasis using a CZT whole-body gamma camera. Eur J Nucl Med Mol Imaging. 2019 Jul;46(8):1672-1677.

17. Melki S, Chawki MB, Marie PY, Imbert L, Verger A. Augmented planar bone scintigraphy obtained from a whole-body SPECT recording of less than 20 min with a high-sensitivity $360^{\circ} \mathrm{CZT}$ camera. Eur J Nucl Med Mol Imaging. 2020 May;47(5):1329-1331.

18. Chevalier E, Boursier C, Claudin M, Marie P-Y, Imbert L. Feasibility of 177Lu Therapy Monitoring Using Fast Whole-Body SPECT Recordings Provided by a High-Speed $360^{\circ} \mathrm{CZT}$ Camera. Clin Nucl Med. 2020;45:e493-4.

19. Bordonne M, Chawki MB, Marie PY, et al. High-quality brain perfusion SPECT images may be achieved with a high-speed recording using $360^{\circ} \mathrm{CZT}$ camera. EJNMMI Phys. 2020 Nov 4;7(1):65.

20. Zeintl J, Vija AH, Yahil A, Hornegger J, Kuwert T. Quantitative accuracy of clinical 99mTc SPECT/CT using ordered-subset expectation maximization with 3-dimensional resolution recovery, attenuation, and scatter correction. J Nucl Med. 2010;51:921-8.

21. Rajasekaran S, Kanna RM, Schnake KJ, et al. Osteoporotic Thoracolumbar Fractures-How Are They Different?-Classification and Treatment Algorithm. J Orthop Trauma. 2017;31:S49-56.

22. Imbert L, Poussier S, Franken PR, et al. Compared performance of high-sensitivity cameras dedicated to myocardial perfusion SPECT: a comprehensive analysis of phantom and human images. J Nucl Med. 2012;53:1897-903.

23. Akamatsu G, Ikari Y, Nishida H, et al. Influence of Statistical Fluctuation on Reproducibility and Accuracy of SUVmax and SUVpeak: A Phantom Study. J Nucl Med Technol. 2015;43:222-6.

24. Lodge MA, Chaudhry MA, Wahl RL. Noise considerations for PET quantification using maximum and peak standardized uptake value. J Nucl Med. 2012;53:1041-7.

25. Bailey DL, Willowson KP. An evidence-based review of quantitative SPECT imaging and potential clinical applications. J Nucl Med. 2013;54:83-9.

26. Dickson J, Ross J, Vöö S. Quantitative SPECT: the time is now. EJNMMI Phys. 2019;6:4.

27. Peters SMB, van der Werf NR, Segbers M, et al. Towards standardization of absolute SPECT/CT quantification: a multi-center and multi-vendor phantom study. EJNMMI Phys. 2019;6:29.

28. Miyaji N, Miwa K, Tokiwa A, et al. Phantom and clinical evaluation of bone SPECT/CT image reconstruction with xSPECT algorithm. EJNMMI Res. 2020;10:71. 
29. Kangasmaa TS, Constable C, Sohlberg AO. Quantitative bone SPECT/CT reconstruction utilizing anatomical information. EJNMMI Phys. 2021;8:2.

30. Delcroix O, Robin P, Gouillou M, et al. A new SPECT/CT reconstruction algorithm: reliability and accuracy in clinical routine for non-oncologic bone diseases. EJNMMI Res. 2018;8:14.

31. Matin P. The appearance of bone scans following fractures, including immediate and long-term studies. J Nucl Med. 1979;20:1227-31.

\section{Figures}
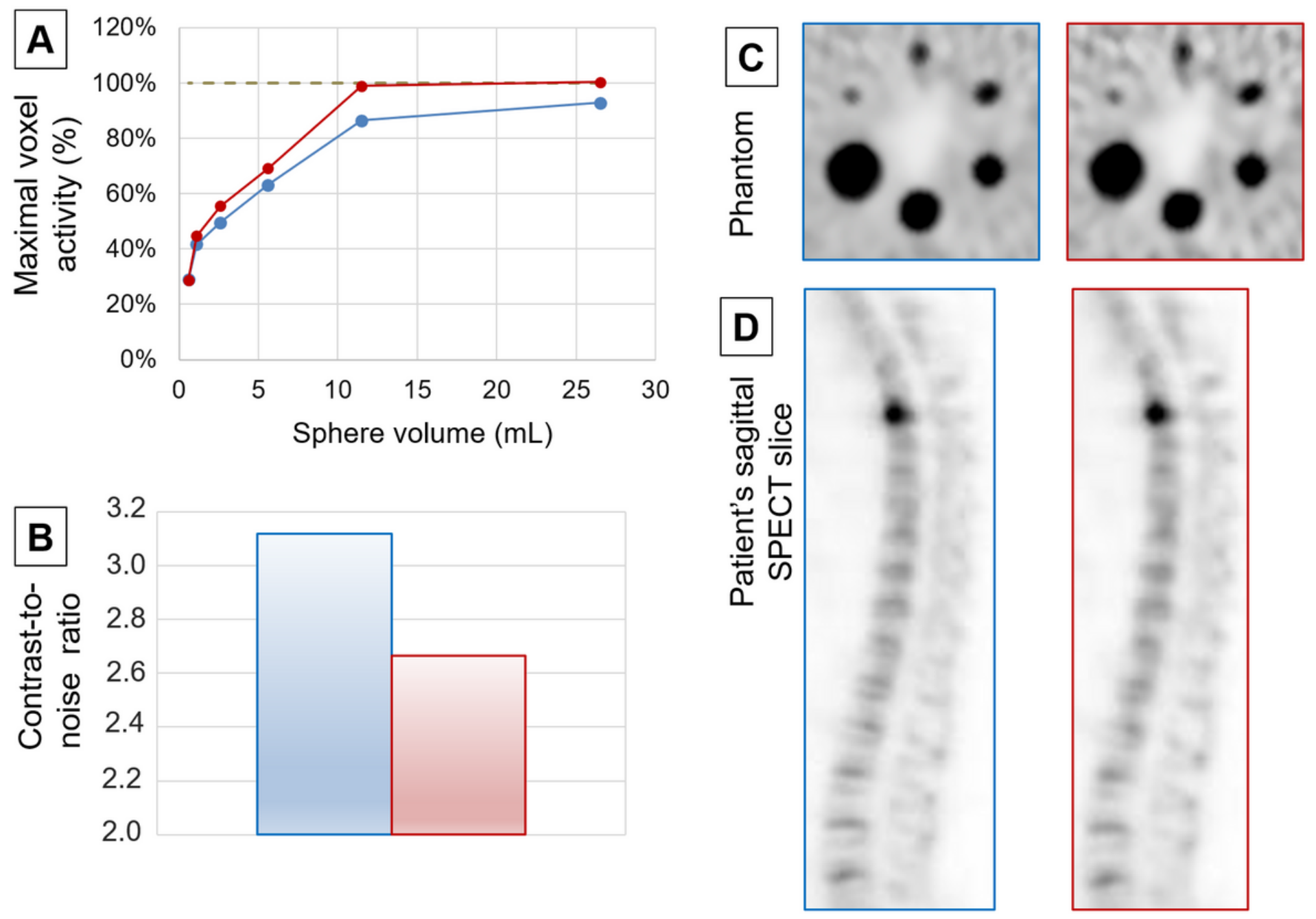

\section{Figure 1}

Comparison of results provided by the 2 recommended OSEM reconstruction processes, the first one favoring spatial resolution but requiring high counts statistics (red lines) and the second one favoring contrast-to-noise ratio (blue lines), with (A) the analyses of the evolutions of the maximal voxel activity concentration measured on the spheres of the IEC phantom and expressed relative to the actual activity concentration, (B) the contrast/noise ratio determined on the IEC phantom, and representative 
tomographic SPECT slices from the IEC phantom and (D) a patient bone sagittal slice passing through a fractured T4 vertebra.

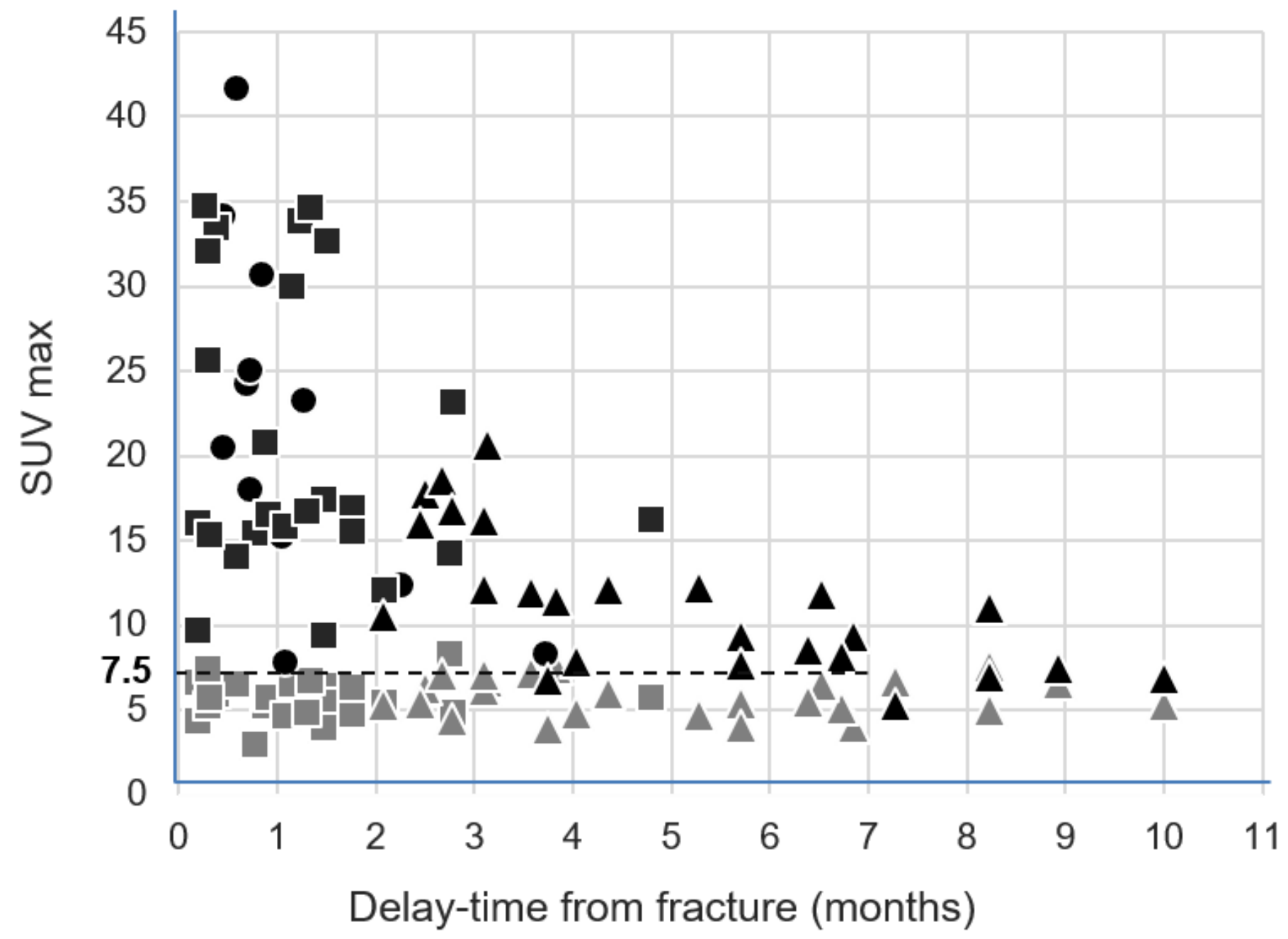

\section{Figure 2}

SUVmax from the 25 intact control T1 vertebrae (grey symbols) and from the 25 initially fractured vertebrae (black symbols) measured on the 1st SPECT/CT (rectangle) and 2nd SPECT/CT (triangle) and displayed according to the delay-time from the date of the initial fracture. The SUVmax from the 12 newly fractured vertebrae apparent on the 2nd SPECT/CT have been additionally inserted according to the delay-time from the corresponding fracture date (black circles). Note that all but one (98\%) of the SUVmax, which were measured on fractured vertebrae up to the 7th month, are $>7.5$, whereas this is the case for only 2 of all SUVmax measurements on the reference intact T1 vertebrae (4\%). See additional comments in the text. 


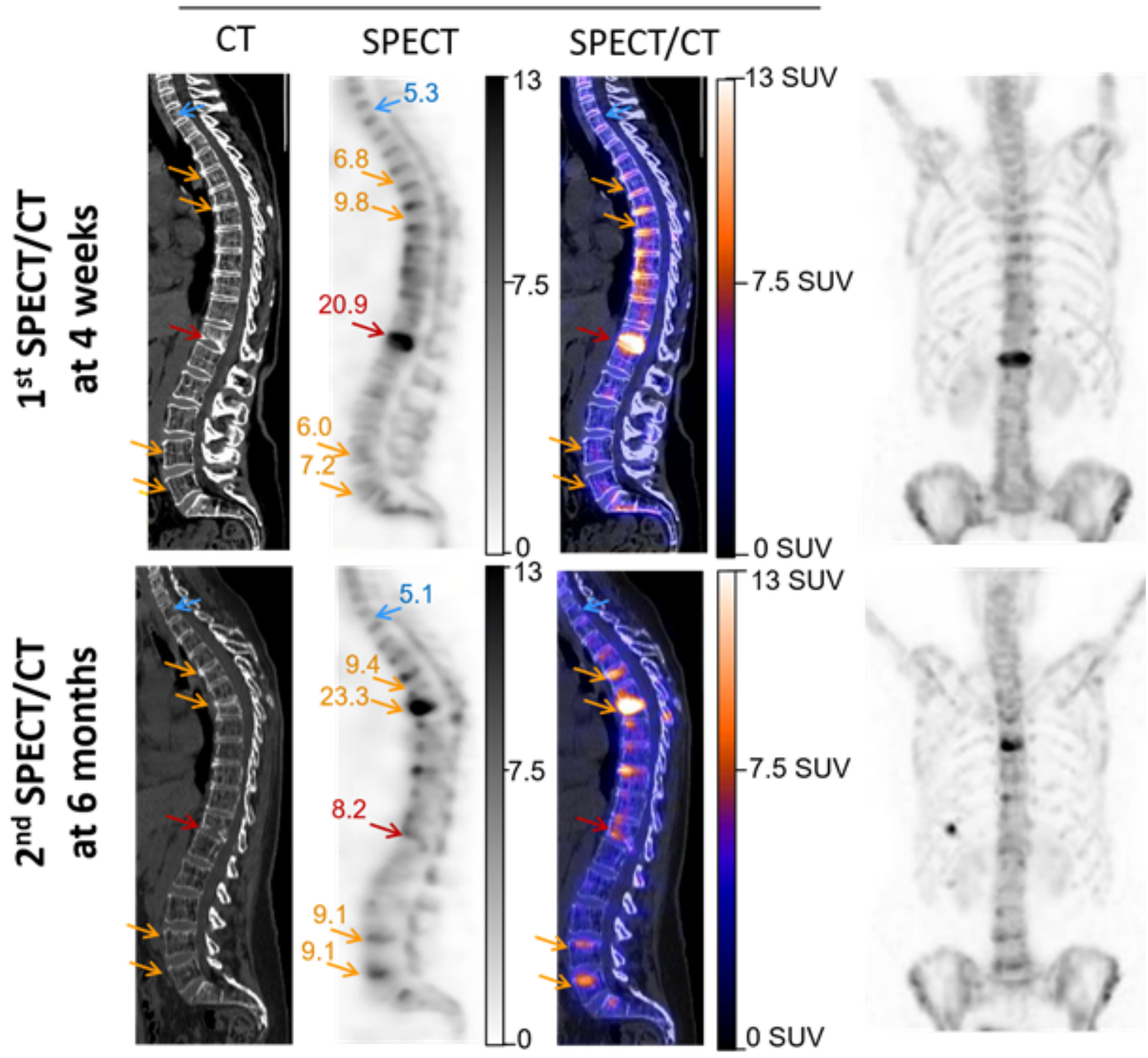

Figure 3

SPECT/CT images recorded in a 56-year-old woman with severe osteoporosis, four weeks after a T12 fracture, and additionally 6-months later, for suspected additional fractures. These latter were finally confirmed on T5, T7, L4, and L5 in a framework of cascade fractures. These images are displayed here through sagittal SPECT, CT, and fused SPECT/CT images, and anterior projections of the maximal intensity projection (MIP) of SPECT images. All SPECT images are similarly scaled from 0 to 13 SUV, thereby facilitating the observations of (i) SUVmax stability between the 2-time points for the reference non-fractured T1 vertebra (blue arrows) and by contrast (ii), SUVmax variations within the fracture sites initially identified on 1st SPECT (red arrows) or newly identified on 2nd SPECT (orange arrows for sites exhibiting significant increases in SUVmax together with CT signs of concomitant compression between the 1st and 2nd SPECT/CT exams). SUV max values have been inserted in the SPECT slices and additional comments are in the text. 


\section{Supplementary Files}

This is a list of supplementary files associated with this preprint. Click to download.

- Suppl.Fig.4.png

- Suppl.Fig.5.png 